\title{
Fault Tolerant Strategies in MANET
}

Ms. Fahmina Taranum ${ }^{1}$, Mr. Khaleel Ur Rahman Khan ${ }^{2}$

${ }^{1}$ Computer Science and Engineering Department, Muffakham Jah College of Engineering and

Technology, Osmania University ${ }^{1}$, , Ace Engineering college ${ }^{2}$, J.N.T.U, Telangana, India,

Email: ftaranum@mjcollege.ac.in ${ }^{1}$ khaleelrkhan@gmail.com

Received: 10 ${ }^{\text {th }}$ Feb 2018, Accepted: $18^{\text {th }}$ March 2018, Published: $30^{\text {th }}$ April 2018

\begin{abstract}
A fault tolerant system for Mobile Adhoc Networks (MANET's) is a system, which works well even in case of failures. The aim of the paper is to handle the issues like network partitioning, cluster head management and data transmission. The parameters like mobility speed, battery value and reach ability range are used for effective implementation. The communication is done via cluster heads with highest mobility speed. Replicas of cluster heads are created for the non-reachable nodes. The number of replicas is equal to double the value of nonreachable nodes. The random values of the replicas for non-reachable cluster head node are better when compared to the old values. The data is send from the sender to the destination node through the cluster heads. The nodes with the three maximum mobility speeds are selected for communication. The data moves from the third maximum mobility speed cluster head node to second and then to the first.
\end{abstract}

Key words: MANETS, TTL, Battery value, Mobility range, Reliability factor.

\section{Introduction}

Mobile ad-hoc networks (MANET's) composed of mobile nodes which can migrate while communicating with each other thereby resulting in partitioning of network. If the node moves within the scope then there will be no network partition. In case of network partitioning there are two cases to consider, one in which the mobile node is a cluster head or simply a communication node. If an unavailable node is cluster head then a new cluster head needs to be elected from the available nodes and when the old cluster head comes back to region of reach ability either it should take over the charge or the new cluster head would retain its charge. This decision has to be taken by the members of the cluster. In the second case if the node is one amongst the node with the three maximum speeds then a replica is created else it would be ignored. Another challenge is to overcome network partitioning. Network partitioning is the condition that exists after a network connection between any two groups of systems fail or a nodes moves out of defined reach ability. When this happen, systems on both side of the apportion can restart their applications resulting in duplicate services. The most serious problem caused by a network partition is that it affects the data on shared storage. Replication is employed as an approach to overcome this problem and to ensure higher service. Replication is widely used for faulttolerance, scalability and performance. The failure of one replica does not stop the system from working as available replicas can take over the tasks of the failed replica. Finally, replication can provide fast local access, even if clients are geographically distributed. Threshold is applied to parameters to declare a node as selfish. Selfish nodes are the nodes participating in the network which hesitate to forward the packets in order to save their resources like low energy constraints.

The general overview and the details of the paper is explained in the following subsections. Section I: Literature Survey, Section II: System design, Section III: Implementation, Section IV: Experimental results and Section V cover Conclusions.

\section{Section-I Related work}

The classification of clustering as local and global was proposed in [1]. Local clusters are created for non- overlapping areas and global for overlapping area. A node with highest battery power and machine load is selected as global cluster leaders. The idea to select the cluster head as a node with the highest battery power is adapted from [1]

[2] Identifies a way to reduce the number of messages required to find a suitable replacement resource among remaining nodes on the network, assuming that redundancy is present among the resources. Our proposed approach works on the suitable replacement of the resource by selecting the top three mobility speed node for data communication and Replication.

Caching effectiveness is calculated as the number of times same document is accessed in [7] Clusters are created by using similar characteristics (viz. nodes at 1 hop distance) and group management by means of cluster head. Using the concept defined in [3], Group communication is implemented by means of cluster head, which interacts with other cluster head in the network and the clusters are created by passing words from a file thereby assigning data to the nodes by taking 
a set of ten nodes in each cluster. The battery value, mobility, range are assigned to the nodes by means of random function and based on defined threshold the nodes are classified as in reach ability or not, cluster head and out of battery in this proposed system.

Being motivated by the relative mobility in [3] and the weighted clustering algorithm concept in [4], an additional parameter Energy is added in collaboration with the range in the proposed model.

Proposed System Model

In this, a general system model is designed to show how the data is communicated in case of failure viz. network partitioning among the members of the clusters. The proposed model consist of Cluster creation module, leader election, Replication and data communication.

Cluster Creation: The clusters are created by passing an input from a file with an extension as .txt, .docx or .doc which reads the words from the file and assign a word to every node. If the number of words in the file is less than the nodes then the duplicate words are assigned to the nodes which are extra else the nodes values are assigned from the words in the file and extra words are ignored. A static function is used to create 5 clusters with 10 nodes in each and the assignment of the values to the node is dynamic, based on the input taken from a file.

Leader election: Leader election is a mechanism used to elect a leader in both wired and wireless systems. For example, in group communication, the election of a new coordinator is required when a group coordinator crashes or departs the system. In the context of wireless networks, leader election is frequently applied because of mobile nodes and dynamically changing topology. Existing solutions to the problem of leader election do not work in the highly dynamic mobile environment. The algorithm used in the proposed system is based on the highest mobility values which represents the speed with which the node moves in the network or the highest battery power. The elected member is selected based on reliability factor (RF).

$\mathrm{RF}=\max$ (Battery) $\| \max$ (Mobility range)

Selfish nodes: Mobile ad hoc networks (MANET) poses new challenges related to security because of relatively different characteristics compared to wired networks. A selfish node is one that tries to utilize the network resources for its own profit but is reluctant to spend its own for others. If such behavior persists among large number of the nodes in the network, it may eventually lead to disruption of network. A selfish Node minimizes efficiency of packet transfer and maximizes the packet delivery time and packet loss rate that divides a network into smaller network. In this paper the selfish nodes are identified as the nodes with the fading battery power and the data is not transmitted via these nodes.

Selfish node $=$ low (mobility value) $\|$ low (battery value)\| low (mobility range)

Replication and network partitioning: Data Replication is a mechanism to enhance data availability. Node are Replicated for increasing the probability of accessing data thereby increasing the throughput and performance of the system. Network partition occurs for out of scope nodes. As the nodes are mobile the possibility of going out of region which is inaccessible increases resulting in network partition. Here the replication is created for those nodes which plays a vital nodes for communication.

No. of Replica $=2 *$ No. of not reachable nodes

Communication Methodology: The data communication is done through cluster head of each clusters as the data is send from source to destination. After division of each node into clusters a proper leader has to be elected as data communication is done via the cluster heads. Leader election algorithm is used to elect a cluster head. Election of leaders must use the highest remaining battery life or the lowest mobility range in the election process. Since nodes with low mobility have least chances of merging and partitioning from their current clusters Types of nodes considered in the communication

1. Green color node in this application is the cluster head.

2. Red color node is the selfish node

3. Blue color node is the node which is out of range.

4. Selfish nodes are determined on the basis of mobility value, battery value and mobility range. If mobility value and battery value of any node reaches a threshold of 5 or less but not zero or if the mobility range is in between 90 to 95 then the node is said to be selfish in this application. A selfish cannot be a leader. Whenever the elected leader is dead or is unavailable multiple replicas of the node are created so as to improve data availability.

\section{PROCESS LOGIC}

A network partition refers to the failure of a network device that causes a network to be split. In this application network partition is said to be occurred, If the mobility range is high (i.e., 
>95) then node is said to be out of range or the battery value is zero or the mobility value is zero (the node is said to be unavailable). The node which is out of the network or which is unavailable is represented with the blue color. For communication the top 3 nodes with the highest values are considered. These nodes are called as active nodes. The data passes from the sender node to leader node in the descending order of the active nodes. The node with the third top most mobility value sends data to the node with the second top most mobility value and the node with the second top most mobility value sends data to the leader node. The leader node sends the data to the leader node of the next cluster. The process continues until the data reaches the receiver node.

\section{Algorithems \\ 1. Leader Election: A leader election algorithm elects a leader from nodes among the clusters considering application-specific conditions.}

Step 0: Parameters used are M, B, R (where M is mobility value or speed of the node, $\mathrm{B}$ is the battery value, \& $\mathrm{R}$ is the mobility range)

Step 1: Set the parameters with Random values

Step 2: Check the battery

value ' $B$ '

Step 3: The node with the highest battery value $B$ is elected as a leader

Step 4: Repeat the process for all the clusters

\section{Network Partition:}

Step 0: Parameters used are M, B, R (where M is mobility value or speed of the node, $\mathrm{B}$ is the battery value, \& $\mathrm{R}$ is the mobility range)

Step 1: Set the parameters with Random values.

Step 2: Check the mobility value ' $\mathrm{M}$ ', battery value ' $B$ ', mobility range ' $R$ '. Step 3:

\begin{tabular}{|l|l|l|}
\hline $\begin{array}{l}\text { Conditi } \\
\text { on }\end{array}$ & Threshold & $\begin{array}{l}\text { Remar } \\
\text { k }\end{array}$ \\
\hline $\begin{array}{l}\text { If the } \\
\text { mobility } \\
\text { range is } \\
\text { high }\end{array}$ & Values $>95$ & $\begin{array}{l}\text { No } \\
\text { de } \\
\text { out } \\
\text { of } \\
\text { ran } \\
\text { ge }\end{array}$ \\
\hline $\begin{array}{l}\text { If } \\
\text { battery } \\
\text { value } \\
\text { or the } \\
\text { mobility } \\
\text { value }\end{array}$ & Value $=0$ & $\begin{array}{l}\text { Networ } \\
\text { k } \\
\text { partitio } \\
\text { n }\end{array}$ \\
\hline
\end{tabular}

Step 4: When network partition occurs, two replicas are created for non-reachable nodes. Step 5: Repeat the process for all the clusters.

\section{Replication:}

Step 0: Parameters used are M, B, R (where $\mathrm{M}$ is mobility, $\mathrm{B}$ is the battery value, \& $\mathrm{R}$ is the mobility range)

Step 1: Set the parameters with Random values.

Step 2: Check the mobility value 'M', battery value ' $B$ ', mobility range ' $R$ '.

Step 3: When network partition occurs, two replicas of each unavailable node are created. The replicated node will have better mobility value and battery values when compared to the original node.

Step 4: Repeat the process for all the clusters.

4. Data Communication:

Step 0: Parameters used are M, B, R (where M is the mobility value, $\mathrm{B}$ is the battery value, $\& \mathrm{R}$ is mobility range)

Step 1: Set the parameters with Random values.

Step 2: Check the mobility value ' $M$ ', battery value ' $B$ ', mobility range ' $R$ '.

Step 3: The data is communicated via the cluster head. Step4: Consider the nodes with the highest

Three Mobility values (say active nodes)

Step 5: Data is passed from the third highest mobility value node to the second highest to the cluster value.

head being holding the maximum mobility

Step 6: Repeat the process for all the clusters until the data reaches the receiver node.

In MANETs, since mobile nodes move freely, network partition may occur, where nodes in one partition cannot access data held by nodes in other partition leading to reduced data availability. Data replication has been widely used to improve data availability, by replicating data at mobile nodes which are not the owners of the original data, data availability can be improved because there are multiple replicas in the network and the probability of finding one copy of the data is higher. The application is based on a replication algorithm which is described for managing replicated files in the case of network partitioning due to node or communication link failures.

\section{Results and Discussion}

The analysis of different scenarios is depicted below to show all possible cases of fault occurring in the system with an option to overcome it by means of multiple strategies defined in the paper. A random function is taken to assign a tooltip with the values of mobility, battery and range. The system works smoothly and the election of leader with highest 
battery power and transmission of data via elected cluster head makes the system more secured and reliable when compared to other approaches defined in research.

Figure 1: Electing efficient leader among the clusters

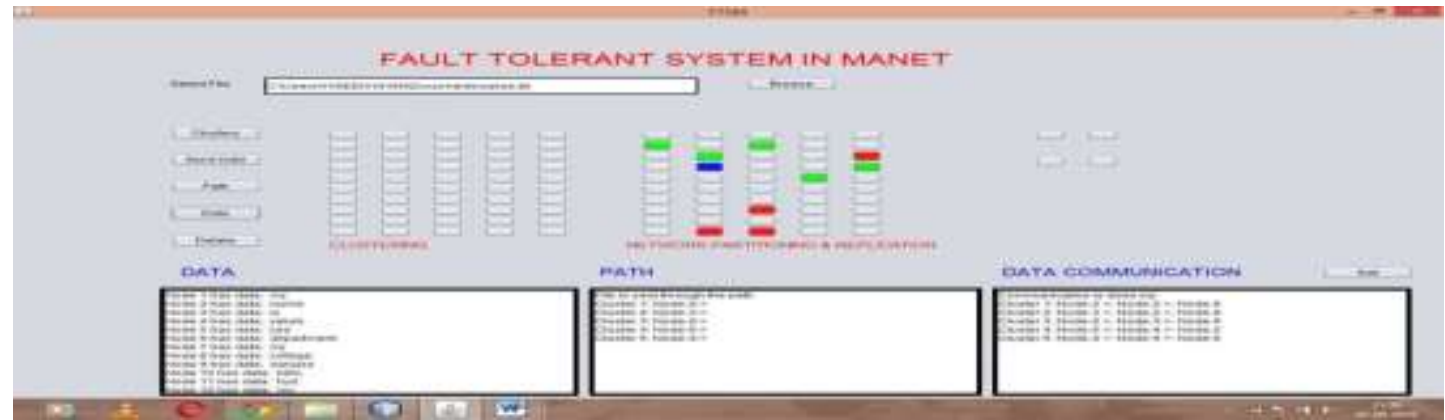

Figure 2: Output of Clustering, Leader Election and Network Partitioning in presence of Selfish Nodes

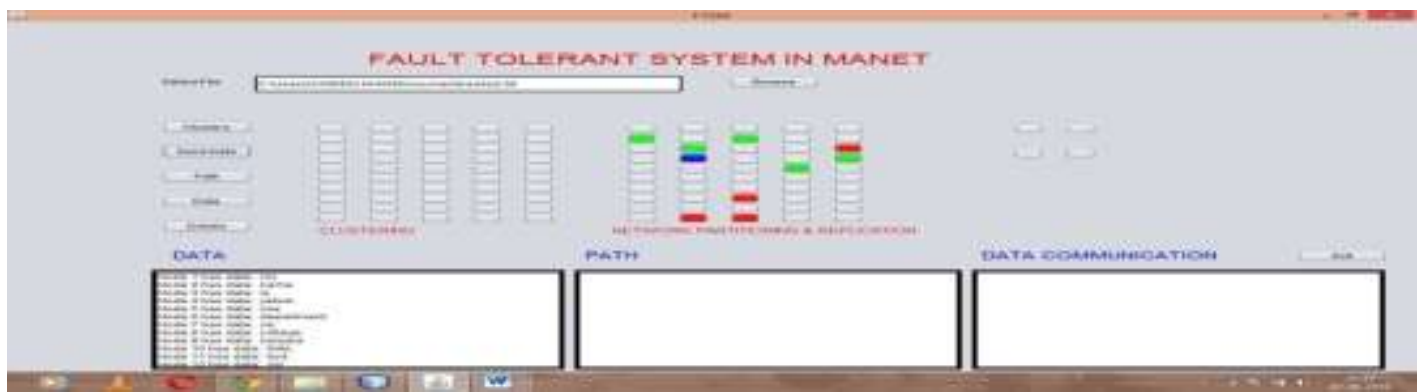

Figure 3: Occurrence of network failure generates replicas for each dead node

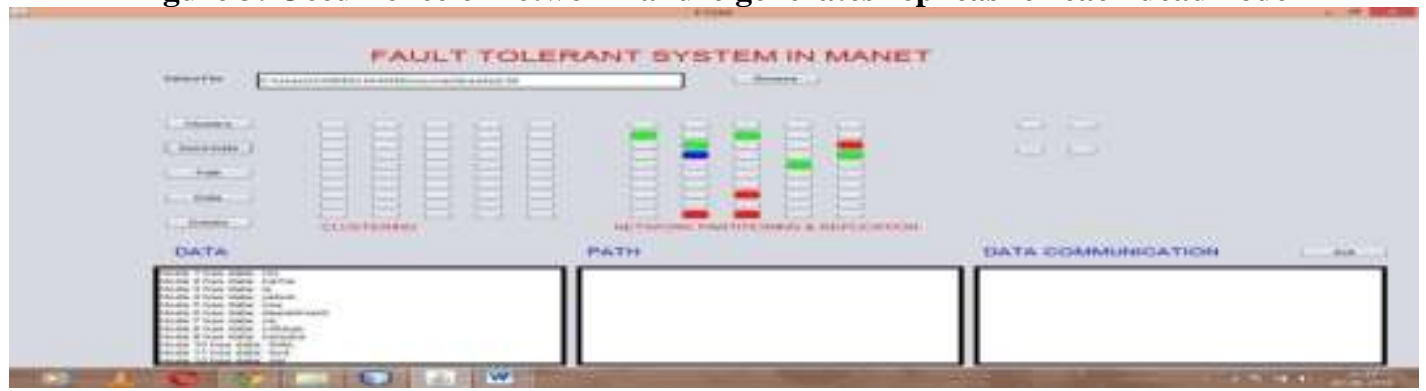

Figure 4: Data Communication via cluster head

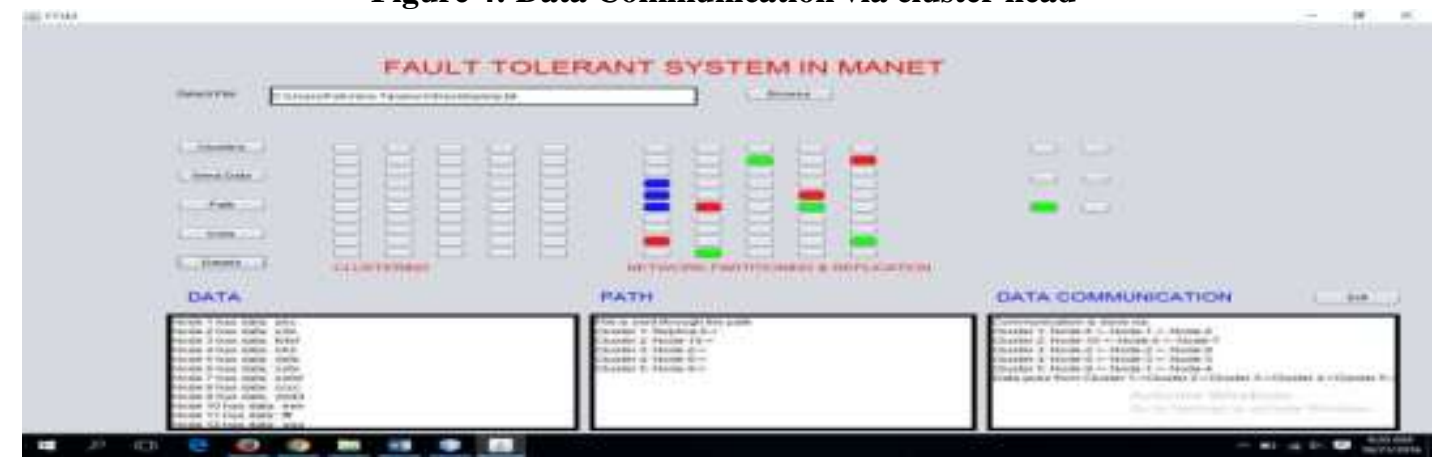


Figure 5: No blue nodes therefore no replica created

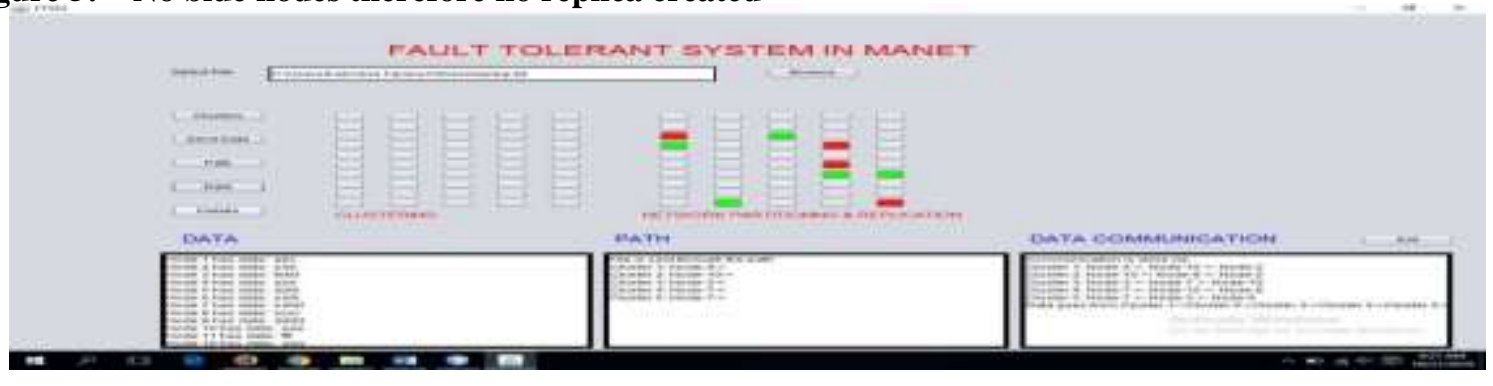

\section{Conclusion}

The proposed approach works well in case of failure of cluster head as the data is passed through the available replica of the cluster head. The system behaves efficiently as the data passes through cluster head always to other cluster head till it reaches the destination node. This application makes a fault tolerant system as it works properly in the presence of a failure node. In this application, clustering is performed where in certain number of nodes are divided into a cluster. Each cluster has a cluster head through which data communication is done. Leader election algorithm is used to elect a cluster head. Election of leaders must use the highest remaining battery life and the lowest mobility range in the election process. Leader election is done in the presence of some selfish nodes, unavailable nodes and nodes which are outside the network. The strategies can be summarized as follows:

1. Solves the network partitioning issue using replication technique

2. The efficient leader is elected in the presence of selfish nodes

3. Maintains replica of the failed node

4. Maintains coordination among the replicas even if the leader node is dead

\section{References}

[1]

bin Paul, k. Preethak; A Cluster Based Leader Election Algorithm for MANETs; International Conference on Control Communication and Computing (ICCC), 2013.

[2]

Adrian P. Lauf, William H. Robinson; Fault Tolerance in MANETs Using a Task-toResource reallocation framework, IEEE 2009 [3]

asu, P., Khan, N., Little, T. D. C.: A Mobility Based Metric for Clustering in Mobile Ad Hoc Networks. In: Proceedings of IEEE ICDC, pp. 413--418 (2001)

[4]

\section{C}

hatterjee, M., Das, S. K., Turgut, D.: WCA: A Weighted Clustering Algorithm for Mobile Ad Hoc Networks. Cluster Computing, 5(2), pp. 193--204 (2002)

[5]

Dav_ce v D. and W. A. Burkhard, "Consistency and Recovery Control for Replicated Files," Proc.10th ACM SOSP, (1985)

[6]

eigenbaum J, S. Shenker; Distributed Algorithmic Mechanism Design: Recent Results and Future Directions; in: Proceedings of the 6th International Workshop on Discrete Algorithms and Methods for Mobile Computing and Communication (Dial- M'02),ACM Press, New York, pp. 1 -13, 2002.

[7]

Komal

M.Sharma and Archana Raut ; Improving the performance of Mobile Adhoc networks using Dynamic Group Data Caching Scheme, IEEE 2013

[8]

Leela

Melit, Nadjib Badache; An Energy Efficient Leader Election Algorithm for Mobile Ad Hoc Networks, Algeria 2014

[9]

Luzi

Anderegg, Stephan Eidenbenz; Ad hoc-VCG: A

Truthful and Cost-Efficient Routing Protocol for Mobile Ad hoc Networks with Selfish Agents; 2011.

[10] The complete reference Java, 7th edition. Herbert Schildt 